\title{
Corneal Injury
}

National Cancer Institute

\section{Source}

National Cancer Institute. Corneal Injury. NCI Thesaurus. Code C35392.

Trauma to the cornea of the eye. 